\title{
Mesomorphic Properties of Side Chain Type Liquid Crystalline Copolyesters with Polar and Non-Polar Groups in the End of the Mesogen
}

\author{
Kohji Ogawa, Takashi Minara, and Naoyuki Koide \\ Department of Chemistry, Faculty of Science, Science University of Tokyo, \\ 1-3 Kagurazaka, Shinjuku-ku, Tokyo 162, Japan
}

(Received July 24, 1996)

\begin{abstract}
Side chain type liquid crystalline copolyesters containing alternative polar and non-polar mesogenic end groups were synthesized. Furthermore, side chain type liquid crystalline copolyester with a random sequence of the polar and non-polar mesogenic end groups was also prepared. The induced smectic phase was exhibited for the copolyester containing alternate polar and non-polar mesogenic end groups in the side chain. The mesomorphic temperature range was influenced by the sequence of the mesogen with the polar or non-polar group. Further, the thermal properties of the mixture of copolyesters with polar and non-polar end groups were investigated. A mixture of $10-50 \mathrm{~mol} \%$ of copolyesters with polar end groups exhibited an induced smectic phase.

KEY WORDS Side Chain Type Liquid Crystalline Polymer / Copolyester / Induced Smectic Phase /
\end{abstract}

It is known that mixtures of nematic liquid crystals having a polar end group like $\mathrm{CN}$ and/or $\mathrm{NO}_{2}$ group with ones containing a non-polar end group such as an alkyl and/or alkyloxy group exhibit induced smectic phases. ${ }^{1}$ Generally, a smectic A phase was induced. Maximal thermal stability of the mesomorphic properties was observed for the 1:1 mixtures of nematic liquid crystals.

In the case of side chain type liquid crystalline polymer (SLCP)s, in general, mesogenic groups were linked to the polymer backbone through flexible spacers. When the polar end group and non-polar end group were introduced to the mesogenic groups in the SLCP, the induced smectic phases were exhibited for the SLCP. Poutugall et al. reported the induced a smectic phase in polyacrylate copolymers with a liquid crystalline side chain containing a polar end group and a non-polar end group. $^{2}$ Further, Imrie et al. reported mesomorphic properties of polystyrenes having azobenzene moieties with a polar end group and a non-polar end group in the side chain. The enhancement of thermal stability for smectic phases in these polystyrenes was observed. ${ }^{3}$

These SLCPs with polymer backbones, namely polyacrylic acid, polymethacrylic acid, and polystyrene were prepared by radical copolymerization of two mesogenic vinyl monomers with a polar end group and a non-polar end group. Therefore, the mesogenic groups would be introduced to the polymer backbone of the SLCP irregularly. Further, the sequence of the mesogenic groups in the SLCPs prepared by radical copolymerization was not clear.

In contrast, polymer backbones like polyurethanes, polyamides, and polyesters have an alternating sequence of two monomers. We are able to recognize the sequence of monomers in the polymer backbone. These polymer backbones with this clear sequence are applicable to SLCPs, especially SLCPs containing mesogenic groups with a polar end group and non-polar end group. Thus, we are able to control the sequence of mesogenic groups in the SLCP.

In this paper, side chain type copolyesters containing mesogenic groups with a polar end group and a non-polar 142 end group, were synthesized and thermal properties for the copolyesters were investigated. Further, in order to clarify the influence of the sequence for mesogenic groups on the thermal properties, random copolyester with an irregular sequence of the mesogenic groups was synthesized. In addition, thermal properties for the mixtures of mesogenic copolyester containing a polar end group were compared with those having a non-polar end group.

\section{EXPERIMENTAL}

\section{Characterization Technique}

${ }^{1} \mathrm{H}-\mathrm{NMR}$ was carried out with a JEOL JPN-PMX60 spectrometer using $\mathrm{CDCl}_{3}$ as the solvent. Infrared spectroscopy data were recorded on a JEOL JIR-7000 spectrometer. Spectra were collected at $4 \mathrm{~cm}^{-1}$ resolution. A minimum of 100 scans was signal averaged. DSC measurements were conducted with a Mettler 3000 series. Wide angle X-ray scattering (WAXS) patterns were recorded on a MAC Science $\mathrm{MXP}^{3} \mathrm{X}$-ray diffractometer equipped with a thermal controller model 5310. Optical microscopy was performed on a Nikon polarizing optical microscopy equipped with a Mettler FP80 controller and a FP82 hot stage. Gel permeation chromatography (GPC) was carried out with a Tosoh HLC-8020 instrument using chloroform as the eluent, equipped with four columns (TSK gel $\mathrm{G} 4000 \mathrm{H}_{\mathrm{HR}}, \mathrm{G} 3000 \mathrm{H}_{\mathrm{HR}}, \mathrm{G} 2000 \mathrm{H}_{\mathrm{HR}}$, and $\mathrm{G} 2000 \mathrm{H}_{\mathrm{HR}}$ ). The instrument was calibrated with a polystyrene standard.

\section{Materials}

Mesogenic diol-monomers and diethylmalonate derivatives were synthesized according to Scheme 1. Side chain type polyesters were synthesized from mesogenic diolmonomers and diethylmalonate derivatives with a polar end group and non-polar one according to Scheme 2.

Diethyl-2-(6-bromohexyl)malonate. In a dry vessel, a dry tetrahydrofuran solution of diethy malonate $(17.8$ $\left.\mathrm{g}, 9.56 \times 10^{-2} \mathrm{~mol}\right)$ and sodium methylate $(28 \%$ in methanol, $27.7 \mathrm{~g}, 0.14 \mathrm{~mol}$ ) was refluxed for 30 minutes. A dry tetrahydrofuran solution of 1,6-dibromohexane 
$(93.3 \mathrm{~g}, 0.382 \mathrm{~mol})$ was added to the mixture and the reaction mixture was refluxed for 4 hours. The filtrate was concentrated and extracted with chloroform. After the chloroform solution was dried over anhydrous magnesium sulfate, the solution was concentrated. The obtained yellow liquid was purified by reduced distillation $\left(1 \mathrm{mmHg}, 135^{\circ} \mathrm{C}\right)$. The product was obtained in a $47.4 \%$ yield.

NMR $\left(\mathrm{CDCl}_{3}\right): \delta 1.2-1.6\left(\mathrm{~m}, 6 \mathrm{H},-\mathrm{COOCH}_{2} \mathrm{CH}_{3}\right)$, $1.6-2.2\left(\mathrm{~m}, 10 \mathrm{H},-\mathrm{CH}_{2}-\right), 3.4(\mathrm{t}, 1 \mathrm{H},-\mathrm{OOCC} \underline{\mathrm{HCOO}}-)$, $3.8\left(\mathrm{~m}, 4 \mathrm{H},-\mathrm{COOC} \underline{\mathrm{H}}_{2}-\right), 4.2\left(\mathrm{t}, 2 \mathrm{H}, \mathrm{Br}-\mathrm{C}_{2_{2}}-\right)$.

Diethyl-2-[6-(4-methoxybiphenyl-4'-oxy)hexyl]malonate. The acetone solution of diethyl-2-(6-bromohexyl)malonate $\left(8.0 \mathrm{~g}, 2.29 \times 10^{-2} \mathrm{~mol}\right)$, 4-methoxy-4'hydroxy biphenyl $\left(4.2 \mathrm{~g}, 2.08 \times 10^{-2} \mathrm{~mol}\right)$, potassium carbonate $\left(11.1 \mathrm{~g}, 8.02 \times 10^{-2} \mathrm{~mol}\right)$, and a small amount of potasium iodide was refluxed for 24 hours. The filtrate was concentrated and the residue was extracted with chloroform. The chloroform solution was dried over anhydrous magnesium. The chloroform solution was evaporated to dryness and the obtained crude white solid was purified by washing with chilled methanol three times. The product was obtained in a $50.2 \%$ yield.

NMR $\left(\mathrm{CDCl}_{3}\right): \delta 1.1-2.2\left(\mathrm{~m}, 16 \mathrm{H},-\mathrm{CH}_{2}-\right.$, $\left.-\mathrm{CH}_{2}-\mathrm{CH}_{3}\right), 3.3(\mathrm{t}, 1 \mathrm{H},-\mathrm{OOCCHCOO}-), 3.7-4.1(\mathrm{~m}$, $\left.9 \mathrm{H},-\mathrm{OCH}_{3}, \mathrm{Ar}-\mathrm{OCH}_{2}-,-\mathrm{COOC} \underline{H}_{2} \mathrm{CH}_{3}\right), 6.7-7.6(\mathrm{~m}$, $8 \mathrm{H}, \mathrm{Ar}-\mathrm{H})$.

IR (Nujol): 1735 (COO), 1606 (Ar).

2-[6-(4-Methoxybiphenyl-4'-oxy)hexyl]-1,3-propanediol. In an atmosphere of nitrogen, a tetrahydrofuran dispersion of $\mathrm{LiAlH}_{4}\left(2.4 \mathrm{~g}, 6.39 \times 10^{-2} \mathrm{~mol}\right)$ was stirred for 30 minutes. To the chilled dispersion, tetrahydrofuran solution of diethyl-[2-(4-methoxybiphenyl-4'-oxy)hexyl]malonate $\left(3.0 \mathrm{~g}, 6.39 \times 10^{-3} \mathrm{~mol}\right)$ was added dropwise. After the reaction mixture had been refluxed for 4 hours, chilled water was added to the reaction mixture. Next hydrochloric acid aqueous solution was added to the mixture, the mixture was concentrated and poured into water. The precipitate was washed with water until a neutral solution was obtained. The white solid was purified by column chromatography (eluent: chloroform). The product was obtained in a $56.9 \%$ yield.

IR (Nujol): 3345 (OH), 1606 (Ar).

Diethyl-2-[6-(4-cyanobiphenyl-4'-oxy)hexyl] malonate. The acetone solution of diethyl-2-(6-bromohexyl)malonate $\left(6.9 \mathrm{~g}, 1.98 \times 10^{-2} \mathrm{~mol}\right)$, 4-hydroxy-4'-cyanobiphenyl $\left(3.5 \mathrm{~g}, 1.80 \times 10^{-2} \mathrm{~mol}\right)$, potassium carbonate $(7.4 \mathrm{~g}$, $\left.5.33 \times 10^{-2} \mathrm{~mol}\right)$, and a small amount of potassium iodide was refluxed for 19 hours. The filtrate was evaporated to dryness and a white solid was obtained. The chloroform solution of the white solid was washed with water. The chloroform solution was dried over anhydrous magnesium sulfate. The filtrate was evaporated to dryness and the obtained solid was purified by recrystallization from methanol. The product was obtained in a $74.3 \%$ yield.

NMR $\left(\mathrm{CDCl}_{3}\right): \delta \quad 1.1-2.2 \quad\left(\mathrm{~m}, 16 \mathrm{H},-\mathrm{C}_{2}-\right.$, $-\mathrm{COOCH}_{2} \mathrm{CH}_{3}$ ), 3.4 (t, 1H, -OOCC $\underline{H C O O}-$ ), 3.7-4.2 $\left(\mathrm{m}, 6 \mathrm{H}, \mathrm{Ar}-\mathrm{OCH}_{2}-,-\mathrm{COOCH}_{2} \mathrm{CH}_{3}\right), 7.0-7.7(\mathrm{~m}, 8 \mathrm{H}$, Ar-H).

IR (Nujol): 2223 (CN), 1716 (COO), 1604 (Ar).

2-[6-(4-Cyanobiphenyl-4'-oxy)hexyl]-1,3-propanediol. In an atmosphere of nitrogen, a tetrahydrofuran solution
$(50 \mathrm{ml})$ of diethyl-2-[6-(4-cyanobiphenyl-4'-oxy)hexyl]malonate and sodium borohydride $\left(2.0 \mathrm{~g}, 5.40 \times 10^{-2}\right.$ mol) was refluxed for 30 minutes. After that methanol $(10 \mathrm{ml})$ was added dropwise to the reaction mixture over one hour. Then the reaction mixture was refluxed for 3 hours. Chilled water and hydrochloric acid aqueous solution were added to the reaction mixture and the solution was concentrated. The residue was poured into water and washed with water until a neutral solution was obtained. The obtained solid was purified by column chromatography (eluent: chloroform). The product was obtained in a $34.3 \%$ yield.

IR (Nujol): 3309 (OH), 2237 (CN), 1606 (Ar).

\section{Polycondensation}

Four alternate copolyesters were synthesized from diethylmalonate derivative and diol-monomer. Polycondensation was carried out using Tetra- $n$-butyl orthotitanate as a catalyst. In a stream of nitrogen, the reaction mixture of diethylmalonate derivative and diolmonomer was heated at $140^{\circ} \mathrm{C}$ for one hour. Then under reduced pressure $(30 \mathrm{mmHg})$, the reaction mixture was heated at $180^{\circ} \mathrm{C}$ for 3 hours. Following the stage, the reaction mixture was then heated at $200^{\circ} \mathrm{C}$ for 5 hours under reduced pressure $(1 \mathrm{mmHg})$. The obtained polyester was purified by dissolving in tetrahydrofuran and reprecipitating with methanol twice. The random copolyester was prepared by polycondensation of two kinds of diol-monomers and two kinds of diethylmalonate derivatives according to the above procedure.

\section{RESULTS AND DISCUSSION}

The routes of synthesis of the diol-monomers are shown in Scheme 1. CN-Diol was prepared by the use of sodium boron hydride as reductant. ${ }^{4}$ The ester groups were selectively converted to the alcohol groups in the reaction. Sodium boron hydride did not influence the cyano groups.

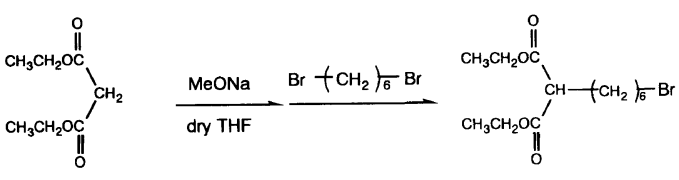

(1)
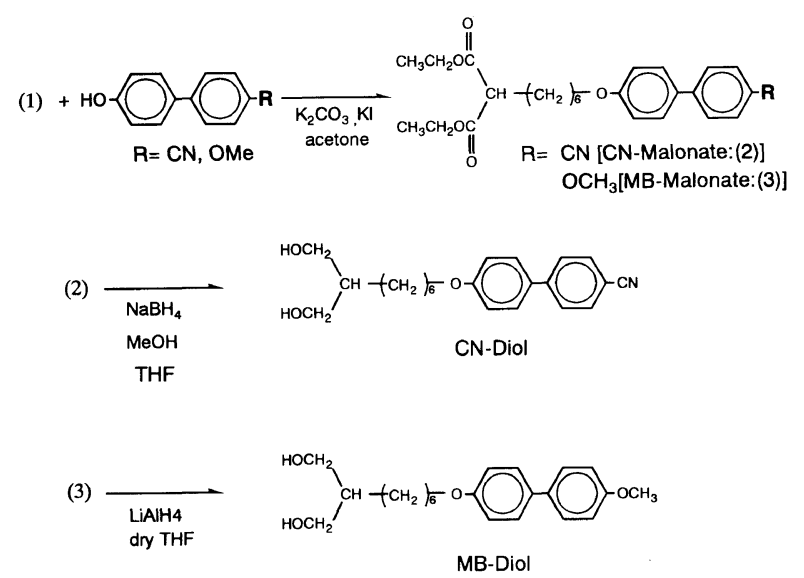

Scheme 1. Synthesis of diethylmalonate derivatives and diolmonomers containing $\mathrm{CN}$ and $\mathrm{OCH}_{3}$ groups at the mesogenic end group. 
Table I. Transition temperatures of diethylmalonate derivatives and mesogenic diol-monomers

\begin{tabular}{lc}
\hline Abbreviation & Phase transition temp $/{ }^{\circ} \mathrm{C}^{\mathrm{a}}$ \\
\hline MB-Malonate & $\mathrm{K} \frac{72}{70} \mathrm{I}$ \\
MB-Diol & $\mathrm{K} \frac{88}{65} \mathrm{M} \frac{138}{133} \mathrm{I}$ \\
CN-Malonate & $\mathrm{K} \frac{63}{?} \mathrm{I}$ \\
CN-Diol & $\mathrm{K} \frac{111}{\frac{\mathrm{M} \frac{}{101} \mathrm{~N}}{108} \mathrm{I}}$ \\
\hline
\end{tabular}

${ }^{\mathrm{a}} \mathrm{K}$, crystal; $\mathrm{N}$, nematic; $\mathrm{M}$, mesophase; $\mathrm{I}$, isotropic.

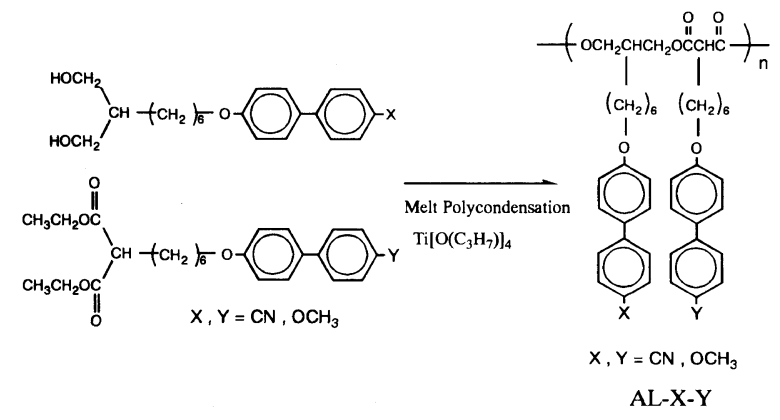

Scheme 2. Synthesis of side chain type liquid crystalline copolyesters.

The thermal properties of malonate derivatives and mesogenic diol-monomers are summarized in Table I. Both diethylmalonate derivatives showed no mesomorphic properties, while mesomorphic properties for both mesogenic diol-monomers were exhibited. The appearance of the mesomorphic properties would be influenced by the hydroxy group generated by the reduction of the ester groups.

MB-Diol demonstrated an enantiotropic mesophase, while a monotropic mesophase was exhibited for $\mathrm{CN}$ Diol. A schlieren texture, characteristic of a nematic phase, was observed on the cooling run for the CN-Diol. Furthermore, another mesophase was observed with decreasing temperature. This mesophase would have a higher order in the structure than that of a nematic phase.

Five kinds of copolyesters were synthesized by melt polycondensation of mesogenic diethylmalonate derivatives and diol monomers (Scheme 2). The thermal properties for the copolyesters are summarized in Table II. AL-MB-MB and AL-CN-CN had the same end group of the mesogen. Both copolyesters displayed an enantiotropic mesophase. In the differential scanning calorimetry (DSC) curve for AL-MB-MB, two peaks were observed on both the heating and cooling runs. One peak was assigned to the melting point, the other one was attributed to the clearing temperature. A focal-conic texture for AL-MB-MB was observed in the temperature range between the melting point and the clearing temperature by use of polarizing optical microscopy measurements, as shown in Figure 1(a). These results indicate that a smectic phase was exhibited for the AL-MB-MB. In the DSC curves for AL-CN-CN, we
Table II. Thermal properties and molecular weight of side chain type liquid crystalline copolyesters

\begin{tabular}{|c|c|c|c|c|c|}
\hline $\begin{array}{c}\text { Abbreviation } \\
(A L \text { or } R A-X-Y)\end{array}$ & & $\begin{array}{l}\text { ase transition } \\
\qquad /{ }^{\circ} \mathrm{C}^{\mathbf{a}}\end{array}$ & temp & $\mathrm{MR} /{ }^{\circ} \mathrm{C}^{\mathrm{b}}$ & $\bar{M}_{n} / 10^{3}$ \\
\hline AL-MB-MB & K & $\frac{127}{123} \dot{S}=$ & $\frac{140}{135} \mathrm{I}$ & 12 & 7.5 \\
\hline \multirow[b]{2}{*}{ AL-MB-CN } & \multirow[b]{2}{*}{$\mathrm{K}=$} & \multicolumn{2}{|l|}{133} & & \\
\hline & & $\overline{127} \mathrm{~s}$ & 135 & 8 & 5.2 \\
\hline \multirow[b]{2}{*}{ AL-CN-MB } & \multirow[b]{2}{*}{$K=$} & \multicolumn{2}{|l|}{130} & & \\
\hline & & $\overline{121} \mathrm{~N}$ & 131 & 10 & 1.9 \\
\hline AL-CN-CN & g & $\stackrel{67}{?} \mathrm{~N}$ & $\frac{115}{115}$ & $48^{\mathrm{c}}$ & 5.1 \\
\hline RA-MB-CN & K & $\frac{106}{100} M=$ & $\frac{163}{159}$ & 59 & 2.0 \\
\hline
\end{tabular}

${ }^{a} \mathrm{~K}$, solid; g, glassy; $\mathrm{N}$, nematic; $\mathrm{S}$, smectic; $\mathrm{M}$, mesophase; I, isotropic. $\quad{ }^{\mathrm{b}} \mathrm{MR}=T_{\mathrm{i}}-T_{\mathrm{m}}\left(T_{\mathrm{g}}\right)$. ${ }^{\mathrm{c}} \mathrm{On}$ heating run.

(a)

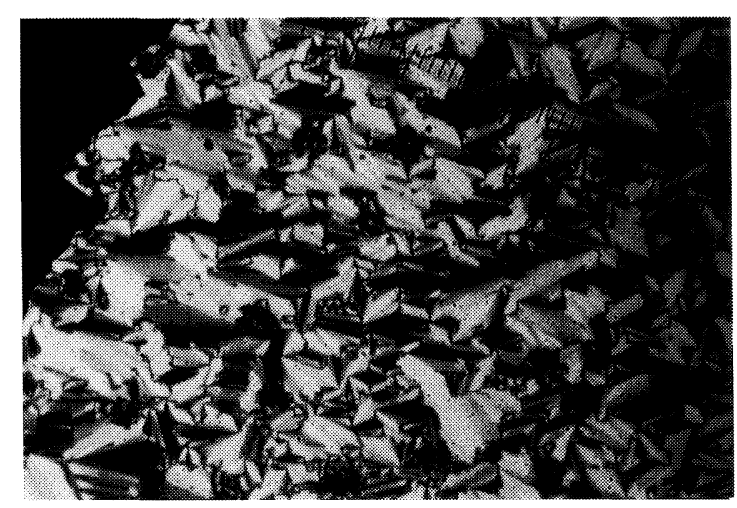

(b)

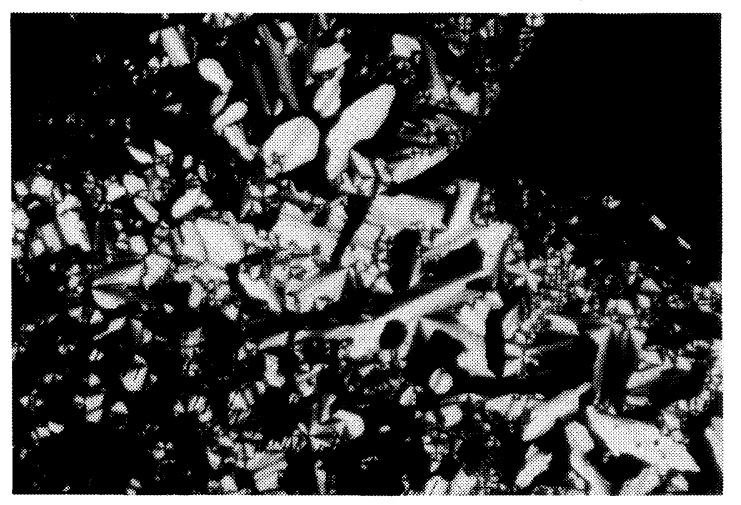

Figure 1. Optical textures of AL-MB-MB at $114.5^{\circ} \mathrm{C}$ (a) and AL-MB-CN at $130.4^{\circ} \mathrm{C}$ (b).

observed a step of the baseline and a peak on both the heating and cooling runs. The step of the baseline corresponded to the glass transition. A peak corresponded to the clearing temperature. A schlieren texture was observed in the temperature range between the two transition temperatures. In the X-ray pattern for the AL-CN-CN, only one broad peak in the wide angle region was observed in the temperature range, as shown in Figure 2(a). These results show that the AL-CN-CN exhibited a nematic phase.

AL-MB-CN and AL-CN-MB had two kinds of 


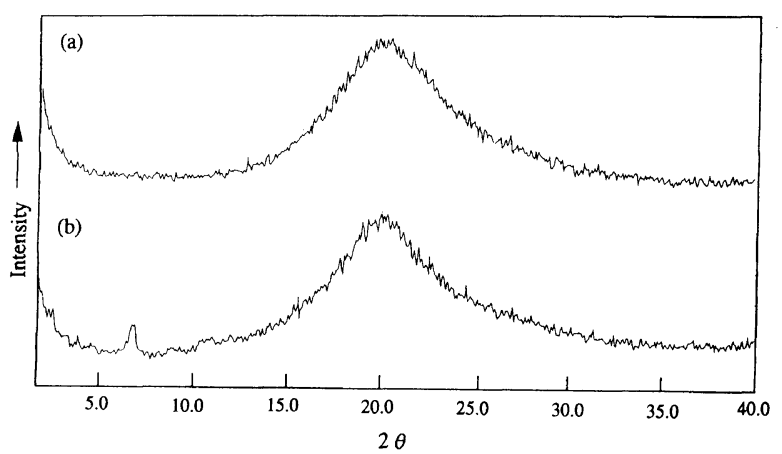

Figure 2. X-Ray diffraction patterns of the copolyesters [AL-CN$\mathrm{CN}$ at $91^{\circ} \mathrm{C}$ (a) and AL-MB-CN at $129^{\circ} \mathrm{C}$ (b)].

mesogenic end group in the side chain. Furthermore, these copolyesters had an alternate sequence of the two mesogenic groups. In the DSC curves of the AL-MB-CN and AL-CN-MB, one peak attributed to the melting point was observed on the heating run, while on the cooling run, two peaks were observed. One peak at the higher temperature was assigned to the clearing temperature and the other one at the lower temperature was attributed to the melting point. The mesomorphic properties were exhibited in the temperature range between the two transition temperatures on the cooling run. In other words, monotropic mesophases were exhibited for the AL-MB-CN and AL-CN-MB, respectively. A focal conic texture was observed for the AL-MB-CN (Figure 1(b)), while a schlieren like texture was exhibited for the AL-CN-MB by use of polarizing optical microscopy measurements in the mesomorphic temperature range. In the X-ray patterns for the AL-MB-CN, a peak in the small angle region and a broad peak were observed in the mesomorphic temperature range, as shown in Figure 2(b). These results showed that the AL-MB-CN displayed a smectic phase. In contrast, in the X-ray patterns for the AL-CN-MB, only one broad peak in the wide angle region was observed in the temperature range. A nematic phase was exhibited for the AL-CN-MB.

RA-MB-CN was also prepared by melt polycondensation of four monomers in the same ratio. The four monomers consisted of the two diol-monomers with methoxy and cyano groups in the end group of the mesogen and the two malonate derivatives. The mesogenic group with the cyano group was introduced to the copolyesters at the ratio of $26 \%$ to all the mesogens. In the DSC curves for RA-MB-CN, two peaks were observed on the both heating and cooling runs. One peak was assigned to the melting point and the other one corresponded to the clearing temperature. A batonnet like texture was observed in the temperature range between the two transition temperatures. We concluded that a mesophase for RA-MB-CN was exhibited from the results of the DSC and polarizing optical microscopy measurements.

Examination of the mesomorphic phase structures for the side chain type liquid crystalline copolyesters revealed that the AL-CN-CN exhibited a nematic phase, but the AL-MB-CN, which had a polar end group and non-polar end group in the end of the mesogen, exhibited a smectic phase. Thus, we concluded that the smectic phase was in fact an induced smectic phase. The layer spacing for

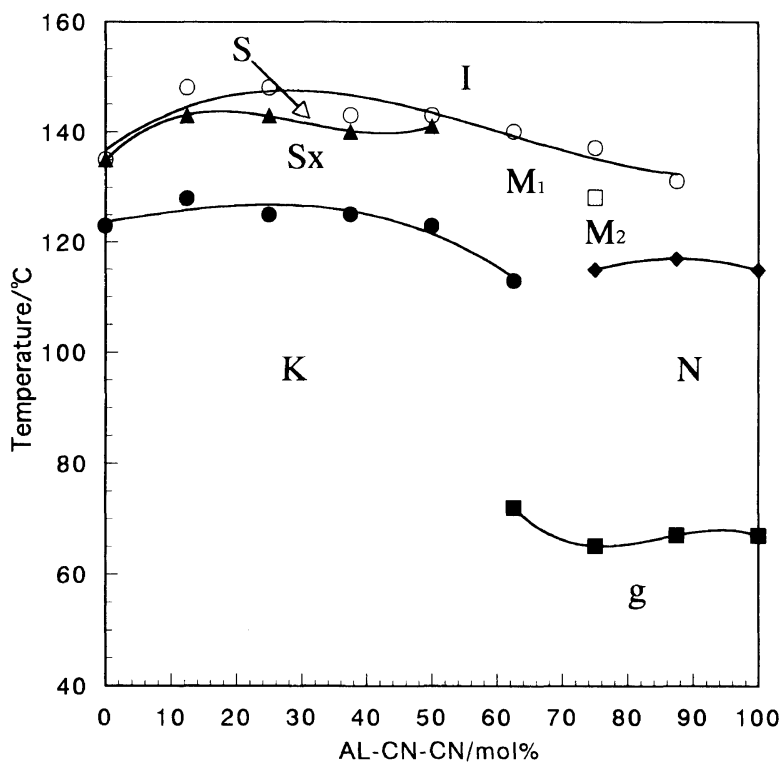

Figure 3. Phase transition temperatures for the mixtures of AL-CN-CN with AL-MB-MB.

the AL-MB-CN was $13 \AA$, while the estimated all-trans molecular length for the side chain was $17 \AA$ for the side chain with the cyano group, and $20 \AA$ for that with the methoxy group. The ratio of the layer spacing to the estimated molecular length was less than 1 . The result showed the phase structure would be the smectic $A_{1}$. We believe that the lateral intramolecular interaction between the polar and non-polar end groups of the mesogen resulted in the smectic phase. The AL-CN-MB had the same end group as the AL-MB-CN, however, a nematic phase was exhibited. The difference in the liquid crystalline properties between the AL-MB-CN and AL-CN-MB, occurred from the structure of the polymer backbone (the direction of ester group) or the molecular weight. Thus, the induced smectic phase was influenced by the structure of the polymer backbone or the molecular weight.

Further, we also paid attention to the mesomorphic temperature range of the copolyesters. In spite of a lower molecular weight, the RA-MB-CN had the largest mesomorphic temperature range of the copolyesters. The RA-MB-CN had the random sequence of the mesogenic group and that of the ester group in the polymer backbone to the polar or non-polar group. This random sequence resulted in the enhancement of the mesomorphic temperature range compared with that of the AL-X-Ys which had alternate and clear chemical structures of the copolyesters.

Further, the thermal properties for the mixture of the AL-MB-MB with the AL-CN-CN was investigated by polarizing optical microscopy and DSC measurements. The thermal properties are shown in Figure 3. The two smectic phases were observed in the content range of $10 \mathrm{~mol} \%$ to $50 \mathrm{~mol} \%$ for the AL-CN-CN. The smectic phase in the higher temperature range corresponded to the induced smectic phase. The textures of the smectic phases are shown in Figure 4. A focal conic fan texture was observed in the induced smectic phase. Furthermore, the focal conic texture was observed with decreasing temperature (Figure 4). On the other hand, a typical 
(a)

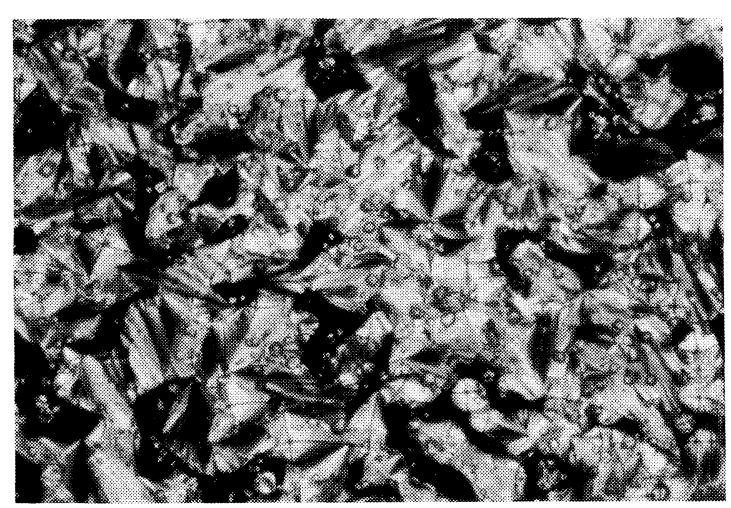

(b)

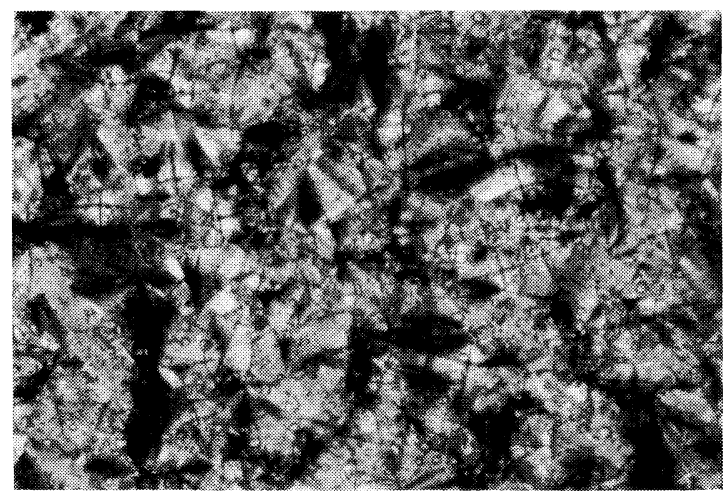

Figure 4. Optical textures of the mixture of AL-MB-MB with AL-CN-CN $(25 \mathrm{~mol} \%)$ at $145.5^{\circ} \mathrm{C}$ (a) and $142.2^{\circ} \mathrm{C}$ (b).

texture was not observed in the content range of $60 \mathrm{~mol} \%$ to $75 \mathrm{~mol} \%$ for the AL-CN-CN. A schlieren texture was observed with increasing content for the AL-CN-CN.

The mesomorphic temperature range for the mixture with the $25 \mathrm{~mol} \%$ of the AL-CN-CN was narrower than that of the RA-MB-CN, which contained the mesogens with the cyano group in the ratio of $26 \mathrm{~mol} \%$. The copolyesters in the polymer mixture contained the same mesogenic groups, whereas the RA-MB-CN had two kinds of mesogenic groups connected to the polymer backbone at random. The difference in the mesomorphic temperature range would occur from the difference in the sequence of the mesogenic group between the RAMB-CN and the copolyesters (AL-MB-MB and AL-CN$\mathrm{CN})$ in the polymer mixture. The mesomorphic temperature range for the polymer mixture containing the AL-CN-CN at the ratio of $50 \mathrm{~mol} \%$ was wider than that of the AL-MB-CN. The AL-MB-CN had the alternate sequence of the mesogenic groups. The high order in the sequence of the mesogenic groups would result in the decrease in the mesomorphic temperature range.

\section{CONCLUSION}

Side chain type liquid crystalline copolyesters containing the alternate sequence of the mesogenic groups with polar and non-polar groups exhibited an induced smectic phase. The induced smectic phases were also observed for the mixtures of the copolymer containing the polar mesogenic end group with the copolymer having a nonpolar one. The mesomorphic temperature range for the copolyesters was influenced by the sequence of the mesogenic groups. With regard to the mesomorphic temperature range, the copolyester with a random sequence of the mesogenic group was superior to the copolyester with alternate sequence of the mesogen and the polymer mixture.

\section{REFERENCES}

1. B. Engelen, G. Heppke, R. Hopf, and F. Schneider, Ann. Phys., 3, 403 (1978).

2. M. Portugall, H. Ringsdorf, and R. Zentel, Makromol. Chem., 183, 2311 (1982).

3. C. T. Imrie, F. E. Karasz, and G. S. Attard, Liq. Crystals, 9(1), 47 (1991).

4. K. Soai, H. Oyamada, M. Takase, and A. Ookawa, Bull. Chem. Soc. Jpn., 57, 1948 (1984). 